\title{
La meditación resulta un tratamiento eficaz para el manejo de algunas variables asociadas con el estrés psicosocial
}

Meditation is an effective treatment for the management of some variables associated with psychosocial stress.

M Goyal y cols. JAMA Intern Med. 2014 Mar;174(3):357-68.

Objetivo

Evaluar el efecto de diferentes programas de meditación sobre distintos aspectos relacionados con el estrés psicológico (ansiedad, depresión, estado de ánimo, calidad de vida relacionada con la salud mental, uso de sustancias, sueño, hábitos alimenticios, dolor y peso).

\section{Fuentes de datos}

Se realizó una búsqueda en MEDLINE, PsycINFO, EMBASE, PsycArticles, Scopus, CINAHL, AMED y la biblioteca Cochrane hasta Junio del 2013.

\section{Selección de estudios}

Ensayos clínicos controlados y aleatorizados que incluyeran adultos $>18$ años con una patología definida, más un estresor. Se incluyeron aquellos trabajos que comparasen programas estructurados de meditación con más de 4 hs de entrenamiento inicial, y además práctica en forma personal, con controles activos (intervenciones equivalentes en tiempo y atención invertida en cada grupo). Éstos, a su vez, se dividieron en controles activos no específicos (ej. programa educativo) y controles específicos. Se excluyeron aquellas prácticas en las que la meditación no es la principal intervención (Yoga, Tai Ji Quan, Biofeedback, Qi Gong, ejercicios de respiración, etc.) y que no tuvieran un control activo.

\section{Métodos}

Se evaluó la calidad de la evidencia en cuatro dimensiones: riesgo de sesgo, consistencia, precisión y objetividad. Se evaluaron diferentes medidas de las intervenciones (dosis de práctica, tipo de práctica, recomendaciones para la práctica individual, adherencia, capacitación del instructor). Se calculó la diferencia relativa de cambio en los puntajes de las escalas utilizadas (puntaje post intervención - puntaje basal grupo intervención) - (puntaje post intervención - puntaje basal grupo control / puntaje basal grupo intervención) como medida de efecto y también meta-análisis de efectos aleatorios teniendo en cuenta precisión, calidad de evidencia, tipo de grupo control y tiempo de seguimiento.

\section{Resultados principales}

Se incluyeron 47 artículos con un total de 3.515 participantes. La tabla 1 muestra los resultados principales. En las comparaciones con controles activos no específicos se encontró una mejoría significativa en variables como dolor, ansiedad y depresión, con moderada calidad de evidencia. También en estrés y calidad de vida, pero con una calidad de evidencia baja. No se observó diferencia significativa en ninguna variable al comparar con controles activos específicos.

Tabla 1: efecto de diferentes programas de meditación sobre distintos aspectos relacionados con el estrés psicológico.

\begin{tabular}{c|c|c|c|c} 
Variable & $\mathbf{n}$ & Total de articulos & Dirección (magnitud) del efecto & Calidad de la evidencia \\
\hline Ansiedad & 647 & 8 & $\uparrow(0 \mathrm{a}+44 \%)$ & Moderada \\
\hline Depresión & 806 & 10 & $\uparrow(-5 \mathrm{a}+52 \%)$ & Moderada \\
\hline Dolor & 341 & 4 & $\uparrow(+5 \%$ a $+31 \%)$ & Moderada \\
\hline Estrés & 735 & 9 & $\uparrow(+1 \%$ a $21 \%)$ & Baja \\
\hline Calidad de vida & 346 & 4 & $\uparrow(+5 \%$ a $28 \%)$ & Baja \\
\hline
\end{tabular}

\section{Conclusión}

Fuente de financiación: Agency for Healthcare Research and Quality (AHRQ).

La meditación resulta un tratamiento eficaz para el manejo de algu-

nas variables asociadas con el estrés psicosocial.

\section{Comentario}

Para el médico de atención primaria es frecuente la consulta por muchos de los síntomas asociados al estrés psicológico que se describen en éste trabajo. Estos cuadros se presentan con toda la complejidad propia de los síndromes relacionados con la salud mental del paciente. La misma se expresa tanto desde la manifestación de síntomas, con la dificultad que esto acarrea para el diagnóstico certero, como en el manejo adecuado y personalizado de los síntomas. Éste meta-análisis presenta la mejor evidencia disponible al momento acerca de la efectividad de la meditación en el alivio de éstos síndromes, siendo un punto fuerte del estudio su estricta comparación contra controles activos. Así y todo, no debemos olvidar las limitaciones metodológicas que presentaron muchos de los estudios incluidos en el meta-análisis (falta de cegamiento en la evaluación de los resultados y en la rama de tratamiento, alta deserción, y falta de análisis por intención de tratar).

\section{Conclusiones del comentador}

Dada la aparente ausencia de daños de la intervención, la debilidad de la evidencia disponible no debería frenarnos a la hora de apoyar a un paciente a iniciar éste tipo de prácticas. 